\title{
Study on the Camouflaged Target Detection Method Based on 3D Convexity
}

\author{
Yuxin Pan \\ Engineering Institute of Engineering Corps, PLA University of Science and Technology, Nanjing 210007, China \\ E-mail:yuxinpan007@163.com \\ Yiwang Chen \\ Ministry of Scientific Research, PLA University of Science and Technology, Nanjing 210007, China \\ Tel: 86-25-8082-0501Ｅ-mail:hailixiansheng@sohu.com \\ Qiang Fu \\ Institute of Science, PLA University of Science and Technology, Nanjing 211101, China \\ E-mail:njfqhp@sohu.com \\ Ping Zhang \& Xin Xu \\ Engineering's Institute of Engineering Corps, PLA University of Science and Technology \\ Nanjing 210007, China \\ E-mail: pinzhangone@yahoo.com.cn
}

Received: June 20, 2011

Accepted: July 7, 2011

doi:10.5539/mas.v5n4p152

\begin{abstract}
To effectively detect the camouflaged target in the complex background, the target detection method based on 3D convexity is proposed in this article. This method uses a new operator which can fully utilize the representative image gray level represented by the convexity structure of the target, set up appropriate threshold to eliminate the influence of the background noise by the median filtering through the gray face of the target image, and realize the effective detection and identification of the convexity target. The experiment result shows that this method could successively detect the camouflaged targets in the complex background, better than classic edge detection method. As a new camouflaged target detection and evaluation technology, this method can provide necessary factors for the design and implementation of the camouflage technology, and promote the development of the camouflage technology.
\end{abstract}

Keywords: Camouflage, Detection, Convexity

\section{Introduction}

The optical camouflage technology usually utilizes various measures to hide, cover, or fade the optical characteristics such as outline and texture of the targets, and make them to be integrated in the background. The usual evaluation method of the effect depends on the manual work to obtain the discovery probability, and this method has certain subjectivity, without strong reliability, such as the Johnson criterion early used by the US army. With the development of the digital image processing technology, some foreign countries begin to use the image characteristics to design, detect, and identify the camouflaged target, and at the same time, the objective analysis and evaluation technology research about the camouflage effect have also been developed. For example, David E. Schmieder et al utilized the contrast ratio of the video image to analyze the relationship between the distinguishability and the discovery probability under different mottled backgrounds, and compared with the result of manual work (David E, Schmieder, 1983, P. 622-630). E. J. Kelman et al studied the body color comparison and vision characteristic of inkfish image, and extracted edges by analyzing the main component quantity of the characteristic, and set them into the chessboard to detect and identify them, and analyzed the effect of the transformed camouflage (E.J.Kelman, 2007, P. 1369-1375). The National Defense Research Center of Denmark developed the camouflage effect simulation evaluation software (CAMEVA) in 1998, and there are several conversion now, and this software extracts the characteristics of the input image, and analyze the statistical result, and in the detector model and the atmosphere transmission model, the detection distance is obtained by the trained minimum scene distinguishability parameter to evaluate the camouflage effect and the detectability of target (Christian M. Birkermark, 1999, P. 229-238), and these researches are mainly centralized in the edge and texture characteristics of the target and background. Li Junwei et al demonstrated a different path, and utilized the difference of the natural background and the fractal characteristic parameter and proposed a kind 
of target detection method combining the fractal technology with the sequence image processing (Li, Junwei, 2003, P. 468-471). However, many complex phenomena contain multiple layers, and each layer has different statistical characteristics, and it is not enough to describe the fractal structure by one parameter, and many parameters are needed to describe the complex phenomena with multiple layers, so Xu Xin et al studied the evaluation of the multi-fractal effect for the camouflage under real and complex background $(\mathrm{Xu}, 2010)$, and some scholars developed the target identification based on the spectrum signature (Anthony A, 2000), and Zhang Chaoyang, Tan Zuojun et al fully recognized the difference of the polarization existing in the scattering of the target and the natural background, and accordingly proposed the camouflage detection technology based on the polarization characteristic (Zhang, 2008, P. 34-37 \& Tan, 2007, P. 200-203), and some literatures also mentioned the biological reference of this method (N. Shashar, 2000, P. 71-75). Though these methods all can detect and identify the camouflage target to some extent, but either the algorithms are not mature, or the application scope is limited.

Unlike above methods, the camouflage target detection method based on 3D convexity is proposed in this article, and there are few researches about this method in China, and this method can effective detect the camouflage target with the convexity characteristics in the complex background, and it is the powerful supplements of existing camouflage target detection methods, and it is very important to improve camouflage design technology and evaluate the camouflaging effect.

\section{The target detection method based on 3D convexity}

Supposed that the input target image is $I(x, y)$, the gradient angle in the Cartesian coordinate system is

$\nabla I(x, y)=\left(\frac{\partial}{\partial x} I(x, y), \frac{\partial}{\partial y} I(x, y)\right)$

, and in the polar coordinate system, the gradient angle $(\theta(x, y))$ can be defined as

$\theta(x, y)=\arg (\nabla I(x, y))=\arctan \left(\frac{\partial}{\partial y} I(x, y), \frac{\partial}{\partial x} I(x, y)\right)$

, and the two-dimensional arc tangent function can be defined as

$$
\arctan (y, x)=\left\{\begin{array}{ccc}
\arctan \left(\frac{y}{x}\right), & \text { if } & x \geq 0 \\
\arctan \left(\frac{y}{x}\right)+\pi, & \text { if } & x<0, y \geq 0 \\
\arctan \left(\frac{y}{x}\right)-\pi, & \text { if } & x<0, y<0
\end{array}\right.
$$

But one-dimensional $\arctan (\mathrm{t})$ is the inverse function of $\tan (\mathrm{t})$, so $\arctan (\mathrm{t}):[-\infty, \infty] \mapsto\left[-\frac{\pi}{2}, \frac{\pi}{2}\right]$. The detection operator based on convexity is noted as $Y_{\mathrm{arg}}$, and it is the partial derivative of the gradient angle by the direction of $y$ is

$Y_{\text {arg }}=\frac{\partial}{\partial y} \theta(x, y)$

Because the paraboloid belongs to random curve, it can be regarded as approximate $3 \mathrm{D}$ convexity or 3D concavity. Supposed that the general equation of the paraboloid is

$f(x, y)=a(x-c)^{2}+b(y-d)^{2}$

, where $\mathrm{a}>0$ and $\mathrm{b}>0$, and its first-order derivatives respectively are

$\frac{\partial}{\partial x} f(x, y)=2 a(x-c)$

$\frac{\partial}{\partial y} f(x, y)=2 b(y-d)$

So its gradient angle is

$\theta(x, y)=\arctan (2 b(y-d), 2 a(x-c))$

And its partial derivative to $\mathrm{y}$ is

$\frac{\partial \theta(x, y)}{\partial y}=\frac{a b(x-c)}{a^{2}(x-c)^{2}+b^{2}(y-d)^{2}}$

Except for the radial $\{(x, y) \mid y=d$ and $x \leq c\}$, this derivative is continuous on the whole plane, and on this radial, its value approaches to infinite, which is denoted by an obvious bright line in the gray scale figure. 
In Figure 1, (a) is the paraboloidal intensity function, $\mathrm{I}(x, y)=5 x^{2}+y^{2}$, and (b) is the gradient argument of (a), and (c) is $Y_{\text {arg }}$ of (a), which has an obviously light line. Because the gradient angle $\theta(x, y)$ is interrupted at the negative semiaxis of $\mathrm{X}$, the intense reaction of $Y_{\text {arg }}$ could be detected at the negative semiaxis of $\mathrm{X}$, which is expressed by that the gradient angle $\theta(x, y)$ sharply changed from the higher limit (about $\pi$ ) to the lower limit (about $-\pi$ ). So at the negative semiaxis of $X$, the partial derivative along the direction of y approaches to infinite, i.e. the value of $Y_{\text {arg }}$ approaches to infinite at the negative semiaxis of $\mathrm{X}$, which can be utilized to detect the zero crossing points of the gradient angle, and these aero crossing points are the grey convexity of the 3D convexity targets in the gray scale. For example, the image of the smooth 3D convexity lambert face (ideal diffuse reflection face) is a convex grey scale function on the point light source. Therefore, the infinite response result detection result of $Y_{\text {arg }}$ is to detect the target which grey face region is 3D convexity.

To avoid the dependence of $Y_{\text {arg }}$ to the convex direction, an isotropous operator can be defined, i.e. the operator which has intensive reaction to all convex directions. The usual method is to turn the original image to the angle $\pi-\alpha$ anticlockwise, and compute the turned image $Y_{\text {arg }}$, and then turn the computed result $Y_{\text {arg }}$ to the original angle ( $\pi-\alpha$ anticlockwise), which is the computation process of image $\alpha_{\text {arg }}$.

Figure 1 (d) is the rotation of (a), calculation of gradient argument, and inverse rotation, and Figure 1 (e) is the rotation of (a), calculation of $Y_{\text {arg }}$, and inverse rotation. Approximately, the isotropous operator $\int_{\alpha=0^{\circ}}^{360^{\circ}}\left(\alpha_{\text {arg }}\right) d \alpha$ can be denoted as

$D_{\text {arg }}=\sum_{\alpha=0^{\circ}, 90^{\circ}, 180^{\circ}, 270^{\circ}} \alpha_{\text {arg }}$

Figure 1 (f) is the response of $D_{\text {arg. }}$. An intense reaction occurs on all axes, and the crossing point of all axes is the peak value point of the values of $D_{\text {arg }}$, i.e. the target point detected by this method.

The operator $D_{\text {arg }}$ is the operator based on the convex structure of the detected target which is the convex points in the target image, and though most backgrounds in the target image are flat, various tiny and local convex elements still exist, and because of the strong reaction of the operator $D_{\text {arg }}$ to the convex characteristic, these convex elements become the noise in the target detection, so it must to pretreat the target image. In the pretreatment of image, only some representative convex faces with bigger distribution regions should be kept, and some small local convexities should be smoothed, and the blurring effect produced because of the smoothing can be ignored basically. For the image smoothing, the smoothing (mean value) filter of the spatial domain is the most effective and simply method, and because some local and tiny convex elements in the target image should be filtered without new extra convexity, the selected filtering masking should be bigger, and the weight coefficients should be equal.

\section{Analysis of the example of the camouflaged target detection method based on 3D convexity}

To test the practicability of the camouflaged target detection method based on 3D convexity, the real example is analyzed in this article. The target image is the camouflaged target imaged collected at the locale, and the program running environment is Matlab 7.0.1.

Experiment approaches:

(1) Collect the camouflaged target images in different environment at the locale, all sizes are $2144 \times 1224$.

(2) Pretreat the images, including the grey scale treatment of images, the gray surface image of image, and the smoothing filtering.

(3) Run the target detection program and find the convex points.

(4) Take the peak value point of $D_{\text {arg }}^{2}$ as the maximum value point, to implement the threshold treatment for the values of $D_{\text {arg }}^{2}$, and find the target points.

(5) Respectively detect the target edges by the representative Sobel operator and the Canny operator.

(6) Experiment result and comparison analysis.

Experiment one:

The target image is the camouflaged soldier with the desert camouflage clothing in the background of withered and yellow grassland.

In Figure 2, (a) is the camouflaged target, (b) is the surface gray figure of (a), (c) is the filtering effect figure of all a matrix of $25 \times 25$, (d) is the computed $D_{\text {arg }}^{2}$, (e) is the $D_{\text {arg }}^{2}$ when the threshold is $90 \%$, and (f) is the final 
detection result.

In Figure 3, (a) is the edge detection result with the Canny operator, and (b) is the detection result with the Sobel operator.

Experiment two:

The target image is the camouflaged soldier with the desert camouflage clothing in the background of green thick growth of grassland.

In Figure 4, (a) is the camouflaged target, (b) is the surface gray figure of (a), (c) is the filtering effect figure of all a matrix of $25 \times 25$, (d) is the computed $D_{\text {arg }}^{2}$, (e) is the $D_{\text {arg }}^{2}$ when the threshold is $90 \%$, and (f) is the final detection result.

In Figure 5, (a) is the edge detection result with the Canny operator, and (b) is the detection result with the Sobel operator.

From above experiment results, it is obvious that the target detection operation based on 3D convexity can effectively detect the camouflaged target in the complex background, but the classic detection operator based on edge cannot detect the target, so this method has the special advantage when detecting the camouflaged target with the convex structure in the complex background. Because the artificial target has may convex elements corresponding to the background, so this operator is the powerful supplements of the detection operator based on edge when detecting and identifying the target.

\section{Conclusions}

In this article, the detection and evaluation method of camouflaged target in the complex background is studied, and the target detection method based on 3D convexity is proposed, and the results of the theoretical analysis and the example computation all show that the target detection method based on 3D convexity could effectively detect the camouflaged target in the complex background, and comparing with the existing edge detection method, this method has obvious advantage, and it can compensate the disadvantages of the existing camouflaged target detecting method, because it considers the relative factors for the design and implementation of the camouflage, and promotes the development of the camouflaging technology. Of course, some problems in this method still have not been solved, for example, the selection of the threshold of $D_{\text {arg }}^{2}$ has not the universality, and will influence the detection result to some extent.

\section{References}

Anthony A.Wasilewski, Nick L.Faust, Theodore J.Doll, et al. (2000). Sensor fusion and image interpretation through integrated spatial/spectral pattern recognition. ADA400210.2000.6.

Christian M. Birkermark. (1999). CAMEVA: a methodology for computerized evaluation of camouflage effectiveness and estimation of target detect ability. SPIE Conference on Targets and Backgrounds: Characterization and Representation V. P. 229-238.

David E, Schmieder. (1983). Detection performance in clutter with variable resolution. IEEE Transactions on Aerospace and Electronic Systems. No. 19(4). P. 622-630.

E.J.Kelman, R.J.Baddeley, A.J.Shohet. (2007). Perception of visual texture and the expression of disruptive camouflage by the Cuttlefish. Sepia Officinalis. Proc.R.Soc. B. No. 274. P. 1369-1375.

Li, Junwei, Zhu, Zhenfu, Jia, Jingcheng et al. (2003). Study on the Target Detection Method Based on the Fractal Technology. Infrared and Laser. No. 32(5). P. 468-471.

N. Shashar, R. Hagan, J.G. Boal, et al. (2000). Cuttlefish use polarization sensitivity in predation on silvery fish. Vision Res. No. 40(1). P. 71-75.

Tan, Zuojun, Li, Jun \& Chen, Haiqing. (2007). Application of the Polarization Detection Technology in the Spatial Target Detection. Laser and Infrared. No. 37(3). P. 200-203.

Xu, Xin. (2010). Study on the Evaluation of the Camouflaged Effect Based on the Multi-Fractal Analysis. Nanjing: Master's Degree Thesis of PLA University of Science and Technology.

Zhang, Chaoyang, Cheng, Haifeng \& Chen, Chaohui et al. (2008). A Study of Polarization Degree and Imaging of Camouflage Net in Natural Background. Journal of National University of Defense Technology. No. 30(5). P. 34-37. 


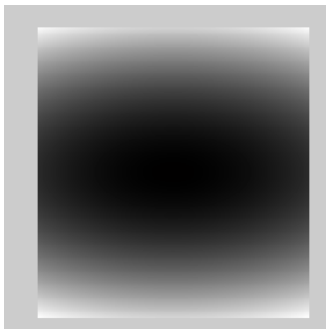

(a)

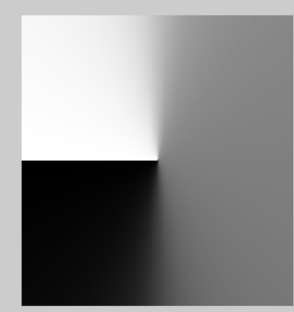

(d)

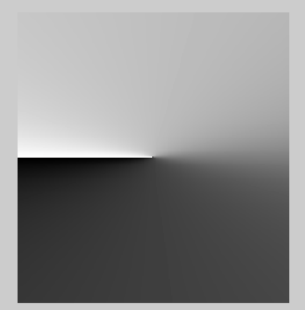

(b)

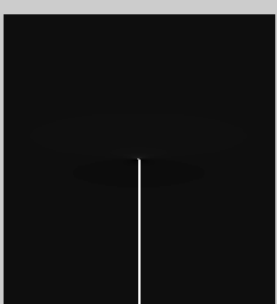

(e)

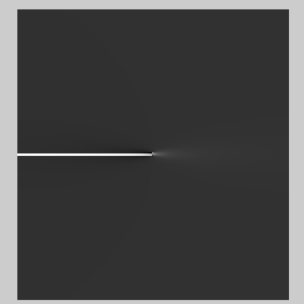

(c)

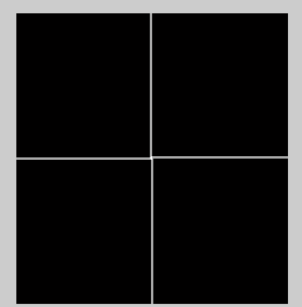

Figure 1. (a) Paraboloidal Intensity Function: $\mathrm{I}(x, y)=5 x^{2}+y^{2}$, (b) Gradient Argument of (a), (c) $Y_{\text {arg }}$ of (a), (d) Rotation of (a), Calculation of Gradient Argument, and Inverse Rotation. (e) Rotation of (a), Calculation of $Y_{\text {arg }}$, and Iinverse Rotation, (f) Response of $D_{a r g}$

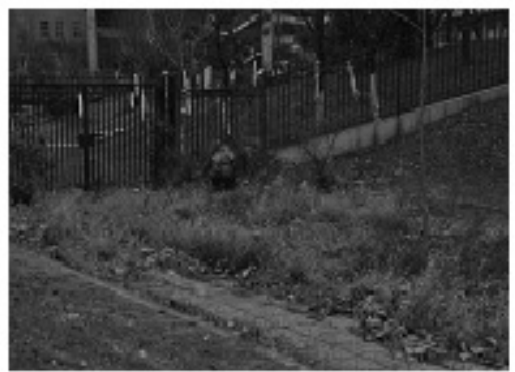

(a)Csmouflaged target

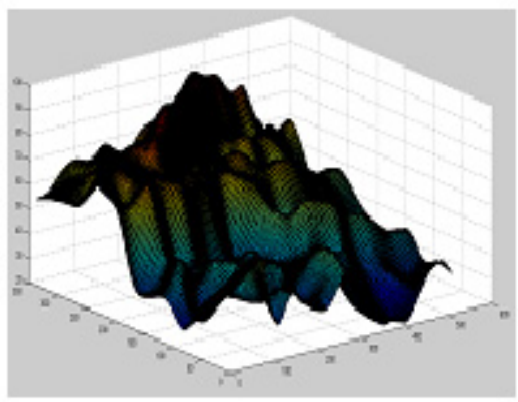

(c) Filtered results

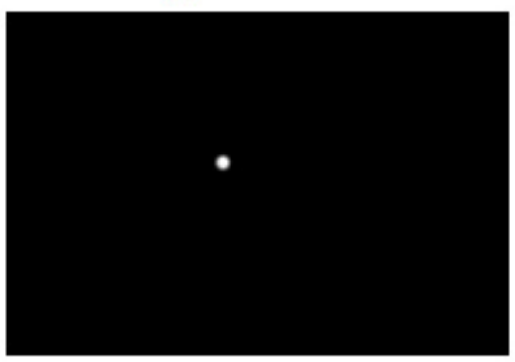

(e) $90 \%$ threshold of Darg

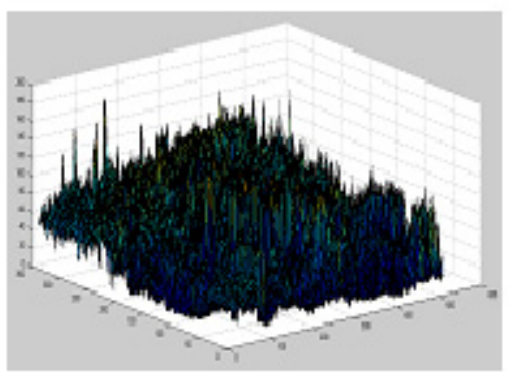

(b)Surface of intensity function

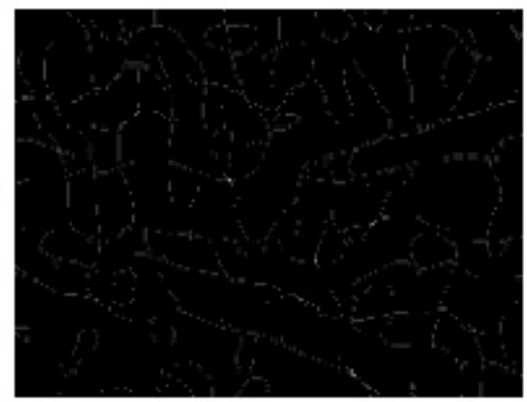

(d) $\operatorname{Darg}^{2}$ of (a)

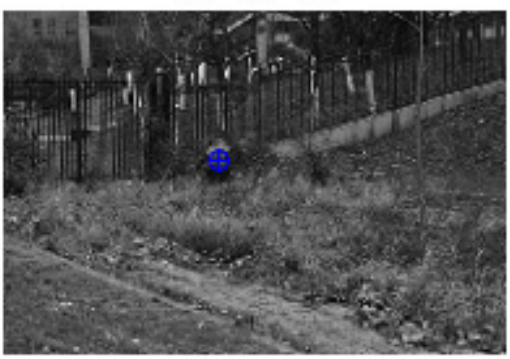

(f) Detection by Darg

Figure 2. Camouflaged Target Detection by Dary Operator 


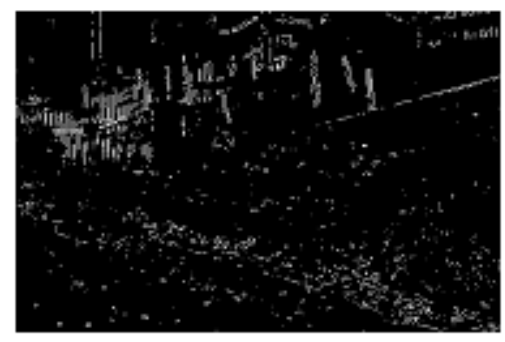

(a)Detection by Sobel

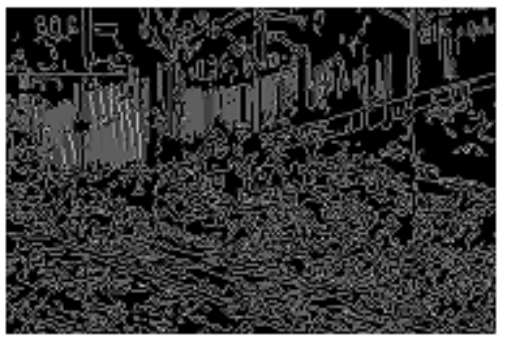

(b)Detection by Canny

Figure 3. Detection Result of the Classic Edge Detection Operator

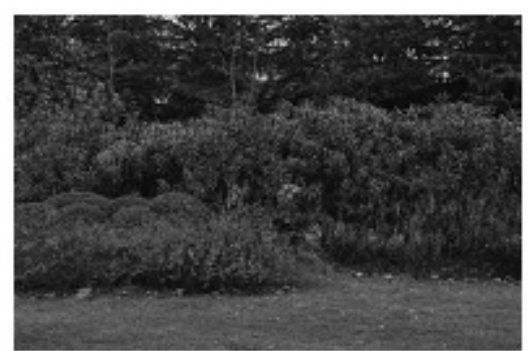

(a) Camouflaged target

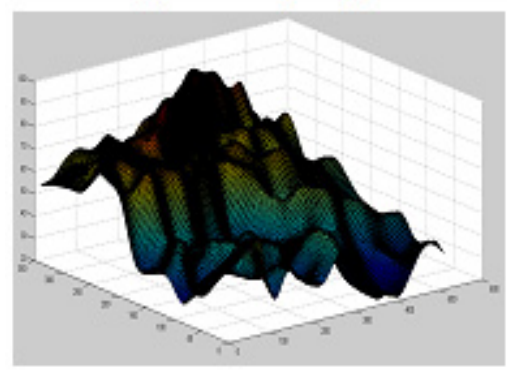

(c) Filtered results

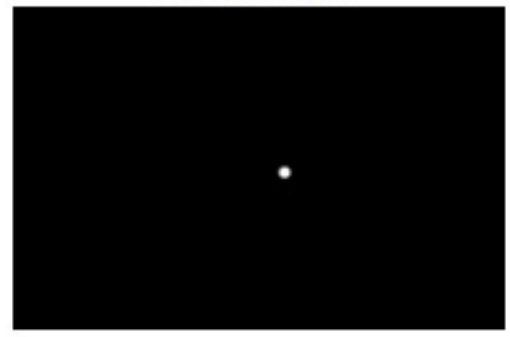

(e) $90 \%$ thre shold of Darg

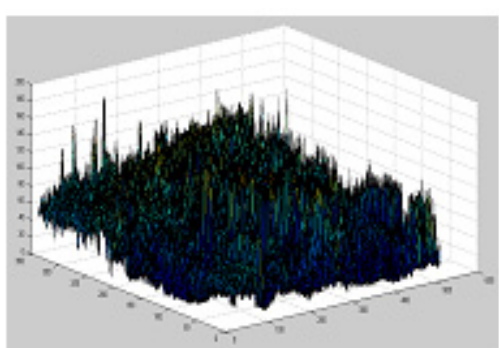

(b) Surface of intensity function

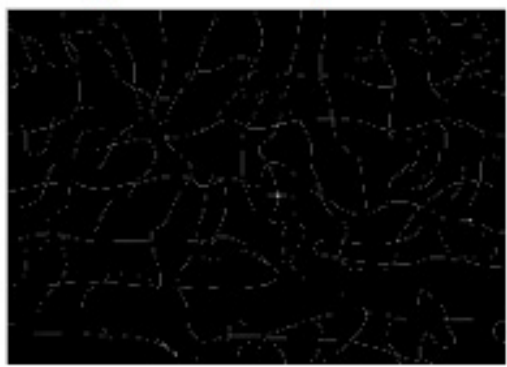

(d) $\operatorname{Darg}^{2}$ of (a)

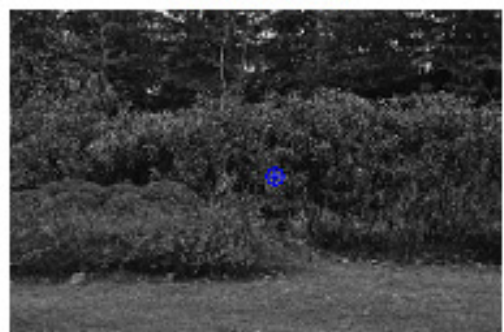

(f) Detection by Darg

Figure 4. Camouflaged Target Detection

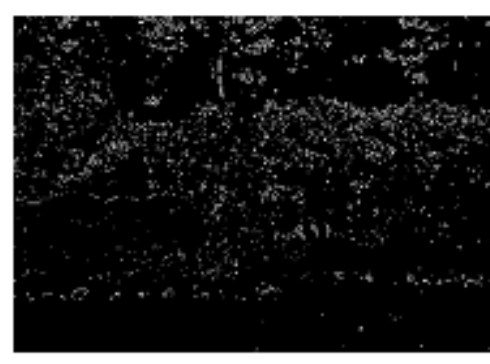

(a)Detection by Sobel

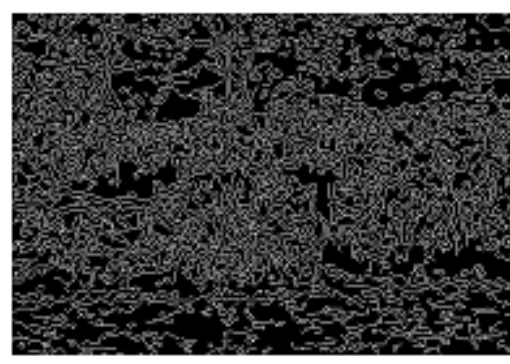

(b) Detection by Canny

Figure 5. Edge-based Detection Result 